\title{
Healthy Eating Behavior Change with Psychological Features and Insulin Resistance: Buffet and Food Diary App Analysis
}

\author{
Meelim Kim \\ Seoul National University College of Medicine \\ Seihee Park \\ Seoul National University College of Medicine \\ Courtney Kim \\ Vanderbilt University College of Arts and Science \\ Hyung Jin Choi ( $\square$ hjchoi@snu.ac.kr) \\ Seoul National University College of Medicine
}

\section{Research Article}

Keywords: eating behavior, psychological features, insulin resistance, buffet test-meal, food diary application

Posted Date: January 13th, 2021

DOI: https://doi.org/10.21203/rs.3.rs-140427/v1

License: (c) (i) This work is licensed under a Creative Commons Attribution 4.0 International License. Read Full License 


\section{Abstract}

Food intake, proportion, and diversity are the major cornerstones of eating behavior. This study examined changes in eating behavior phenotypes using a randomized controlled study (RCT) of digital cognitive behavioral therapy for healthy behavior augmentation (dCBT), with 45 individuals in the dCBT group and 25 individuals in the control group. The dCBT group received a daily intervention for lifestyle modification, while the control group performed self-care for eight weeks. The food intake, proportion, and diversity of both groups were assessed using two different methods: a food diary via a mobile app and buffet test meals consisting of 24 food items classified as healthy or unhealthy. Results revealed that dCBT was successful in promoting healthy eating behaviors that led to physiological and psychological adjustment for the metabolic mechanisms and consequences of healthy eating behavior. Restrained eating behavior at baseline significantly predicted changes in food intake and diversity of healthy diets after the intervention. Lastly, changes in satisfaction with body shape and insulin resistance were significantly correlated with changes in food intake and diversity in healthy diets. These findings suggest that investigating eating behaviors using objective and self-report methods and psychological and physiological indices can facilitate individualized treatment in obesity clinics.

\section{Introduction}

The prevalence of obesity has remarkably increased over the past decades, making obesity the most critical public health issue. Although the etiology of this condition is complicated, maladaptive eating behavior is acknowledged to be the major contributor ${ }^{1}$. Thus, eating behaviors are significant considerations of the public health burden ${ }^{2}$. There is growing interest in research on eating behaviors due to their importance in obesity. Previous studies have examined three different dimensions of eating behaviors: food intake, proportion, and diversity ${ }^{3-5}$. Food intake refers to the amount of food consumed. This is the most remarkable eating behavior phenotype, since an energy imbalance, the main feature of overweight condition and obesity, occurs when the intake exceeds the expenditure ${ }^{5}$. Food proportion refers to the percentage of food group sections composing the total food intake ${ }^{6}$. There are controversial results regarding the relationship between food proportion and bodyweight, showing all three possible outcomes (positive, negative, and no significant associations) $)^{1,2,5}$. Food diversity is related to the distribution or diversity of dietary patterns among different food groups ${ }^{4}$. Although few studies have investigated the role of food diversity in health outcomes, there is an increasing recognition that food diversity can be particularly relevant to obesity and blood glucose control in diabetes ${ }^{3,6}$. Moreover, it may be a principal indicator for evaluating the nutritional status of the diet as a whole. A comprehensive understanding of eating behavior requires the consideration of multifaceted factors. Therefore, understanding the mechanisms for controlling human eating behaviors and developing the ability to assess them is critical in establishing effective obesity treatments.

From a psychological perspective, there are three fundamental questions related to studying human eating behaviors: How much do we eat (food intake)? How much do we choose to eat from specific food groups (food proportion)? How diverse are the categories of the food we eat (food diversity)? There is no doubt that emotions and eating behaviors interact with each other. For instance, emotion could influence food intake and choice by altering appetite or food availability, while emotional transitions can also result from food intake and choice ${ }^{7}$. In addition, people regulate eating behaviors according to contextual aspects such as social settings ${ }^{5}$, food variety ${ }^{8}$, food palatability ${ }^{9}$, memory of recent eating ${ }^{10}$, and motives for eating regulation ${ }^{11}$. The great majority of research on eating behaviors within psychology is about managing food intake, choice, and variety, since these can be associated with obesity and eating disorders.

One of the important factors in eating behaviors is insulin resistance (IR), a dysmetabolic status in which the peptide hormone insulin shows reduced biological effect on peripheral target tissues. Previous studies have revealed that IR is closely related to obesity, metabolic diseases such as type 2 diabetes (T2D) mellitus and cardiovascular disease, and cognitive impairment. IR is associated with the central nervous system delivering information regarding energy homeostasis and is positively associated with adiposity ${ }^{12,13}$. A previous study also reported that IR patterns correlated positively with eating behaviors such as overeating and food craving, potentially stimulating altered regulation of the homeostatic system ${ }^{14}$. Accordingly, reducing the food calorie intake decreases both body weight and IR ${ }^{15,16}$. In further human brain studies, insulin activation selectively impaired the prefrontal cortex and hypothalamus of overweight and obese people $\mathrm{e}^{14,17}$. However, the role of IR during changes in eating behavior, food intake, proportion, and diversity for weight loss has not been comprehensively investigated. 
To comprehend human eating behaviors, it is critical to establish assessment methods for analyzing food intake, proportion, and diversity ${ }^{18}$. Since the nature of eating behavior is complicated, an optimal protocol for its assessment has not yet been defined. In fact, current protocols to analyze eating behavior lack precision and have systematic limitations. Some past studies insisted upon approaches that consider both internal validity (reproducibility) and external validity (resemblance to real-world eating behavior) ${ }^{19}$. Thus, a comprehensive assessment indicating ideal circumstances (high internal validity) and real-world conditions (high external validity) will promote the efficacy and safety of new medical interventions for obesity using randomized controlled trials (RCTs) ${ }^{20}$.

Among the protocols focused on internal validity (reproducibility), many techniques and methodologies have been developed based on experimental conditions of food intake, proportion, and diversity. The most common method involves allowing participants to take meals with no restrictions on the amount of food ${ }^{19}$. This method has shown good validation for total energy intake between two equal sessions, presenting good reproducibility 21,22 . The internal validity of buffet test-meals in laboratory research is generally high because they provide the maximum sensitivity level and allow for manipulation of the intervention and the outcomes ${ }^{19}$. It is also practical to categorize food into either healthy or unhealthy groups based on a structured procedure. However, under these conditions, there is a high risk of excluding the cognitive and physiological aspects of eating behavior.

Besides the buffet test-meal, several studies have highlighted the importance of considering the real-world eating context, focusing on external validity (resemblance to real-world eating behavior) ${ }^{23,24}$. Most recent research has emphasized using digital technologies such as mobile apps to aid dietary assessment ${ }^{25}$. Food diaries using mobile apps enable the use of advanced technological features, such as wireless communications and portable designs, allowing people to reflect real-life eating behavior and situations in dietary research. Thus, these apps are suitable for determining eating behavior at breakfast, lunch, dinner, and snacks, representing daily patterns. While the external validity of mobile apps for assessing eating behaviors is high, several methodological limitations threaten internal validity ${ }^{19}$. The most severe problem is that the chance of data collection errors is relatively high, usually due to misreporting of energy intake. However, little research has examined the validity of logging food intake and choice through mobile apps.

This study explores the role of psychological characteristics and IR in eating behaviors by implementing a buffet test-meal (experimental setting) and a food diary using a mobile application (real-world setting) to develop precision medicine in obesity clinics. The predictors and mechanisms related to eating behavior changes based on lifestyle modification clinical trial data (shown in Fig. 1) were investigated.

\section{Methods}

\section{Study design and participants}

This investigation followed up on findings initially addressed in a digital cognitive behavioral therapy (dCBT) study ${ }^{26}$. It was found that dCBT successfully induced lifestyle modifications, thereby supporting a healthy weight. The data supporting the aim of the present analysis are from the previous dCBT study. This study was an 8-week randomized controlled trial with active dCBT intervention. The trial was registered with ClinicalTrials.gov NCT03465306 in 18 September 2017, https://register.clinicaltrials.gov/NCT03465306. The detailed design and methods have been published elsewhere ${ }^{26}$. Briefly, the 70 subjects in this study (all female) were randomly assigned to a control (app only) group or a dCBT (app + human CBT) group at a ratio of 1 to 2 . The dCBT group received daily feedback and assignments from a psychologist based on the CBT modules for eight weeks; members could access the digital tools from the intervention period through the 24-week follow-up. The control group was instructed to use only a food diary without therapist intervention until the 24-week follow-up, but was given the same digital tools and instruction as the dCBT group. The daily feedback and tasks based on the CBT modules were delivered to the dCBT group by a psychologist for eight weeks. The control group, provided with the same digital devices and guidance as the dCBT group, was instructed to use only a food diary without therapist intervention.

\section{Assessment}

\section{Procedures of the buffet test-meals and food diary apps}


This study consisted of a breakfast session that took place between 8:00 a.m. and 10:00 a.m. It was composed of 24 food items (12 food items for a healthy diet and 12 food items for an unhealthy diet; Appendix A, Table A1). Healthy diet foods have low calorie density and glycemic index (GI), while unhealthy diet foods have relatively high calorie density and $\mathrm{Gl}^{27}$. Prior to the meal, the participants were given an explanation about the foods available at the test-meal and told they could decide which foods to eat, and as much or as little as they wanted. All participants had 30 minutes to eat, but they were not required to eat for this entire time if they finished earlier. The majority of the participants consumed their meals within 15-20 minutes. They were also informed that they could ask for additional servings of any of the foods provided. During the meal, the total weight of each food consumed was measured from pre- to post-meal, and nutrient values were calculated using nutrition facts label information to determine total meal intake. Participants were also instructed to log their food diaries using the Noom app during the 8-week active intervention. Noom consists of a personalized self-monitoring system designed to record meals, exercise, and weight. The food diary provides nutritional information for each food based on the food database.

Food intake is the quantity of food consumed by the subject. The food intake phenotypes were composed of nine indices for the buffet test-meal method: total calories (FIB-T), caloric intake of healthy diet foods (FIB-H), that of unhealthy diet foods (FIB-UH), the intake amount of carbohydrate (FIB-Carb), protein (FIB-Pro), fat (FIB-Fat), sugar (FIB-Su), sodium (FIB-So), and saturated fat (FIB-Sf), and nine indices for the food diary in the app: total calories (FID-T), the amount of carbohydrates (FID-Carb), protein (FIDPro), fat (FID-Fat), sodium (FID-So), and calorie intake for breakfast (FID-B), lunch (FID-L), dinner (FID-D), and snacks (FID-S). For example, the intake amount of carbohydrate (FIB-Carb) is the absolute value of the total amount of carbohydrate intake during the buffet meal-test.

Food proportion is defined as the proportion of each food type (healthy/unhealthy or carbohydrate/protein/fat) consumed by the subject, composing the total food intake. The food proportion phenotypes consisted of five markers for buffet test-meal assessment: the proportion of chosen amounts from highly healthy diet foods (FPB-H), unhealthy diet foods (FPB-UH), carbohydrate (FPB-Carb), protein (FPB-Pro), and fat (FPB-Fat); and three markers for the food diary in the app assessment: carbohydrate (FPD-Carb), protein (FPD-Pro), and fat (FPD-Fat). For example, the healthy diet proportion score (FPB-H, 0-100\%) = $[($ amount of healthy diet food intake) / (amount of total dietary intake) $\times 100$; unhealthy diet proportion score $(\mathrm{FPB}-\mathrm{UH}, 0-100 \%)=$ [(amount of unhealthy diet food intake) / (amount of total dietary intake)] $\times 100$.

Food diversity is the diversity of dietary patterns chosen by the subject among the buffet food item groups served; it is not the same as the proportion of foods consumed. Food diversity phenotypes refer to the number of food categories consumed from a previously selected list. Thus, these categories were derived from the buffet test-meal method only and defined as follows: total food diversity score $($ FDB-T, $0-100 \%)=[$ (the number of food items consumed from all 24 food items provided) $/ 24] \times 100$; healthy diet diversity score $(\mathrm{FDB}-\mathrm{H}, 0-100 \%)=[$ (the number of food items consumed from the 12 healthy food items provided) $/ 12] \times$ 100 , and unhealthy diet diversity score (FDB-UH, 0-100\%) $=$ [(the number of food items consumed from the 12 unhealthy food items provided) / 12] × 100. The classifications of the overall eating behavior index are presented in Appendix A (Table A2).

\section{Other baseline assessment (anthropometrics, psychological, and biological measures)}

Anthropometric measures, including body composition, such as whole-body fat mass, fat percentage, and lean body mass, were measured using a portable bioelectrical impedance analysis device (InBody H20B; InBody Co., Ltd, Seoul, South Korea). After overnight fasting, blood samples were taken to measure glucose, triglyceride, total cholesterol, gamma-glutamyl transpeptidase, insulin, and leptin in the morning to avoid daily variations in activities. A revised version of the Situational Motivation Scale was used to assess the participants' situational motivation toward the behavioral change to lose weight. In addition, the psychological measures were assessed using a brief form of the Body Shape Questionnaire-8C (BSQ-8C), the Korean version of the Beck's Depression Inventory (K-BDI-II), the Trait Anxiety Inventory, the Rosenberg Self-Esteem Scale, the Dutch Eating Behavior Questionnaire (DEBQ), and the Automatic Thoughts Questionnaire (ATQ-30). All these questionnaires were in Korean, and detailed explanations can be found elsewhere ${ }^{26}$.

Intervention 
A multi-dimensional, daily individualized intervention was delivered by a psychologist using CBT modules via the mobile app. CBT contents are focused on promoting the eating behavior of healthy foods based on published programs guided by clinicians ${ }^{28}$. This is different from other behavioral modification programs that encourage dietary restraints on eating habits ${ }^{29,30}$. Its contents instruct people to stop skipping meals and eat breakfast by conforming to healthy behavioral habits. Accordingly, the diverse components relevant to each participant's behavior, cognition, emotion, and motivation in the dCBT group were observed and examined during the 8-week active intervention. A detailed description of the intervention procedures is provided by Kim et al. ${ }^{26}$

\section{Statistical analysis}

The mean and SD were used to describe baseline characteristics. Differences between the two groups were detected using an independent-samples $t$-test for continuous variables and a chi-square test for dichotomous variables. A paired-samples $t$-test was conducted to examine the statistical differences between baseline and post-intervention within a group. Effect sizes were also estimated using Cohen's $d$. Correlation analysis using Pearson's coefficient was used to investigate which baseline measures had a predictive role in food choice and food intake changes at eight weeks. The coefficient of variation (CV) was used to determine the standardized measure of distribution dispersion. All analyses were conducted using SPSS Statistics software, version 25 (IBM Corp.), and two-tailed statistical significance was set at $p=.05$. As there were less than $20 \%$ missing values in the set of overall changes variables, mean imputation was performed.

\section{Results}

Table 1 shows all participants' baseline characteristics in both the dCBT and control groups; details have been published previously ${ }^{26}$. 
Table 1

Baseline characteristics of participants in both groups.

\begin{tabular}{|c|c|c|}
\hline Characteristics & Control $(n=25)$ & Digital CBT $(n=45)$ \\
\hline Age, years, mean (SD) & $21(2.7)$ & $22.3(3.5)$ \\
\hline Fasting glucose, $\mathrm{mg} / \mathrm{dL}$, mean (SD) & $87(8.1)$ & $87.3(7.4)$ \\
\hline Triglyceride, mg/dL, mean (SD) & $92.2(35.9)$ & $93.2(42.6)$ \\
\hline Total cholesterol, $\mathrm{mg} / \mathrm{dL}$, mean (SD) & $184.7(24.9)$ & $191.1(30.4)$ \\
\hline GGT, U/L, mean (SD) & $15.3(8.5)$ & $21.3(32.8)$ \\
\hline Leptin, ng/ml, mean (SD) & $37.5(14.7)$ & $42.49(15.3)$ \\
\hline Fasting insulin, $\mu \mathrm{U} / \mathrm{mL}$, mean (SD) & $12.6(6.1)$ & $16.1(9.1)$ \\
\hline SIMS, score, mean (SD) & $77(5.8)$ & $76.1(5.7)$ \\
\hline BSQ-8C, score, mean (SD) & $34.8(8.9)$ & $36.24(7.5)$ \\
\hline K-BDI-II, score, mean (SD) & $14.7(9.6)$ & $13.6(9)$ \\
\hline TAl, score, mean (SD) & $47.8(11)$ & $48(10.4)$ \\
\hline RSES, score, mean (SD) & $21.9(6.4)$ & $19.8(5.6)$ \\
\hline DEBQ-restrained score, mean (SD) & $30.6(7.3)$ & $29.9(6.6)$ \\
\hline DEBQ-emotional score, mean (SD)* & $29.1(11.6)$ & $38(10.1)$ \\
\hline DEBQ-external score, mean (SD)* & $32(7)$ & $34.9(4.8)$ \\
\hline ATQ-30, score, mean (SD) & $57.6(26)$ & $57.2(22.3)$ \\
\hline
\end{tabular}

\section{Changes in eating behavior phenotypes (treatment efficacy)}

Figure 2 shows each behavioral phenotype's overall results for both healthy and unhealthy diets from the buffet test-meal assessment. Regarding eating behavior phenotypes from the buffet test-meal, participants in the dCBT group showed a significant change in FIB-H (Cohen's $d=0.60$, dCBT: $M=26.06, S D=68.95$ vs. Control: $M=-8.00, S D=46.41 ; p=.03$ ) and FDB-H (Cohen's $d=$ 0.66 , dCBT: $M=4.62, S D=11.54$ vs. Control: $M=-2.78, S D=10.85 ; p=.01)$ at eight weeks compared to the control group. For the eating behavior phenotypes from food diary data, the changes in mean FID-B (Cohen's $d=0.60$, dCBT: $M=26.75, S D=154.56$ vs. Control: $M=-41.63, S D=46.98 ; p=.03$ ) of the dCBT group were significant compared to the control group at eight weeks. No significant differences were observed in other eating behavior phenotypes (Appendix A, Table A3 and A4).

In addition, only the dCBT group showed significant changes in the FIB-H (Cohen's $d=0.73, M=135.33, S D=185.70 ; p<0.001$ ), FPB-UH (Cohen's $d=0.54, M=13.98, S D=25.87 ; p=0.003$ ), and FDB-H (Cohen's $d=-0.35, M=-4.66, S D=13.32 ; p=.049$ ) after the 8-week intervention. The dCBT and control groups showed significant changes in FIB-UH, FPB-H, FDB-UH, and FDB-T.

According to the eating behavior phenotypes from the food diary assessment in the app, only the dCBT group showed significant decreases in both the FID-L (Cohen's $d=-0.39, \mathrm{M}=-68.85, \mathrm{SD}=174.90 ; p=.008$ ) and FID-D (Cohen's $d=-0.43, \mathrm{M}=-111.97$, SD $=257.86 ; p=.005$ ) at eight weeks compared to the baseline. Both groups achieved significant FID-S changes in the last week compared to the first week (dCBT: Cohen's $d=-0.62, M=-73.32, S D=117.74 ; p<.001$ vs. Control: Cohen's $d=-0.59, M=-$ 124.26, $S D=211.07 ; p=.006$ ). The overall results of calorie intake per meal were reported in a previous study ${ }^{26}$. Figure 3 shows the results of food intake changes per meal assessed by the food diary in the app (Appendix A, Table A5 and A6).

\section{Predictors and mechanisms related to eating behavior change}


Restrained eating behavior, measured by the DEBQ (DEBQ-RE) at baseline, was significantly correlated with FDB-H change at eight weeks $(p=.006)$. Participants who had greater dietary restraint intention or behavior at baseline were more likely to increase the number of food categories among the healthy diet foods after the 8-week intervention. The change in the level of body shape satisfaction, assessed by the BSQ-8C during the 8-week intervention, showed a significant positive correlation with FIB-H change $(p=.039)$ and FDB-H change $(p=.04)$. The calorie intake and number of healthy diet food categories increased as the level of body shape satisfaction increased. The change in IR (fasting insulin) was also significantly correlated with FIB-H change ( $p$ $=.043)$ and FDB-H change $(p=.026)$. Thus, participants with greater calorie intake and more categories among healthy diet foods showed a greater decrease in IR during the 8-week intervention. Other parameters showed significant or meaningful results. Figure 4 illustrates the significant correlations between the predictive markers and the mechanisms related to the changes in major eating behavior phenotypes. All results of the correlation analysis are presented in detail in Appendix A (Table A2).

\section{Functional relationship between the two assessments of eating behavior}

Considering the CV among the two different assessments, the CV of FIB-T (46\%) was greater than that of FID-T (28\%) after dCBT. The CV of these two assessments was similar to other nutritional indices such as carbohydrate (FIB-Carb vs. FID-Carb; $45 \%$ vs. $34 \%$ ), protein ( $52 \%$ vs. $41 \%)$, fat ( $77 \%$ vs. $45 \%)$, and sodium ( $59 \%$ vs. $47 \%)$. In addition, there was no significant correlation between FIB-T and FID-T or among other indices after dCBT $(p=.806)$.

\section{Discussion}

This study successfully demonstrated the function of psychological features and IR concerning changes in healthy diet eating behaviors. Food intake and diversity were promoted after the lifestyle modification intervention. Baseline psychological characteristics effectively predicted these changes in eating behaviors. In addition, changes in both psychological conditions and IR could explain the changes in eating behavior. This study also demonstrated for the first time the usefulness of implementing two different assessment methods for eating behaviors: the buffet test-meal and food diary in the app.

\section{Food intake, proportion, and diversity change via dCBT intervention}

To our knowledge, this is the first study to investigate changes in food intake, proportion, and diversity obtained through dCBT in an RCT design. These changes were implemented by comparing the dCBT group to the active comparators applied in the control group. Regarding the experimental setting measures, FIB-H and FDB-H significantly improved in the dCBT group compared to the control group. In addition, according to the measures in the real-world setting, the change in FID-B significantly increased in the dCBT group compared to the control group. Thus, dCBT successfully improved both food intake and diversity in healthy diets and encouraged people to eat breakfast.

\section{Methodology: multi-dimensional eating behavior}

For the first time, this study assessed eating behavior with multi-dimensional (intake, proportion, diversity, healthy vs. unhealthy diet foods, nutrients) phenotypes in a prospective study design. Several previous studies have used cross-sectional research designs or a 24 -h food recall method ${ }^{18}$ with only a unidimensional index to assess eating behavior ${ }^{31,32}$. For this reason, they cannot provide correlational and comprehensive explanations of changes in eating behaviors. In contrast, this study observed behavioral changes in multiple aspects of eating behavior, such as food intake, food proportion, and food diversity. Specifically, the food diversity index is applicable when there are more than four different dietary categories ${ }^{18}$. The study developed the most effective design to assess food diversity behavior by applying the most diverse numbers of food items within the 24 food items. Moreover, it provides evidence of causal relationships among eating behaviors, psychological features, and physiological factors due to the prospective design. Thus, this study investigates the multiple dimensions and mechanisms of eating behavior, dissecting its inherent complexities.

\section{Methodology: buffet test-meal and food diary in app}

This is also the first study to assess eating behavior with two different procedures: a buffet test-meal (experimental setting) and a food diary using a mobile app (real-world setting). Previous studies assessed eating behavior by applying only a single method, either an experimental test meal ${ }^{33}$ or a food diary $\mathrm{app}^{34}$. Studies assessing eating behavior with laboratory meals showed higher 
reliability and validity, but insufficiently reflected real-world eating behaviors. In contrast, those using food diary apps were more likely to reflect the real-world setting. However, they showed underreporting of the measures related to the smartphone app's ability, the level of engagement in the app, and either overestimated or underestimated records provided by self-report assessment ${ }^{34,35}$. Based on the results of the study, the behavioral eating patterns detected by the two assessment methods showed differences in performance. The $\mathrm{CV}$ of the buffet test-meal was greater than that of the food diary app. This result could be interpreted to indicate that the buffet test-meal effectively demonstrates the actual heterogeneity of individuals' eating behavior characteristics.

Compared to the app's food diary (12 indices), the buffet test-meal (17 indices) provided a higher eating behavior index. In addition, buffet-test meals defined the nutritional components and identified healthy or unhealthy foods. We successfully detected changes in eating behavior related to healthy diets, but did not find a significant change among the nutritional factors. This may be because the main goal of dCBT was to promote a healthy diet rather than control nutritional factors. The food diary app could specify each diet and the daily meals, such as breakfast, lunch, dinner, and snacks, differently from the buffet test-meal. This study successfully detected a significant change in breakfast intake that provides further evidence to support the idea that the main result of the dCBT intervention was to enhance a healthy diet change.

Taken together, these results support the idea that the buffet test meal is more relevant to quantifying the eating behavior changes after the intervention, while the food diary app has the advantage of identifying breakfast, lunch, and dinner meals separately.

\section{Psychological features predicting healthy eating behavior improvement}

Although several previous studies examined the predictors of weight loss ${ }^{26,36}$, this study is the first to identify predictors for changes in eating behaviors during a lifestyle modification intervention. We found that a restrained eating behavior style was the most favorable predictive marker of healthy eating behavior among individuals in the dCBT program. This result provides new evidence that the dCBT program could be most beneficial to improving the diversity in a healthy diet for people who have a restrained eating behavior style.

Indeed, restrained eating behavior is a barrier to successful weight loss due to loss of control of eating in response to certain events such as emotional stress, alcohol, or palatable foods ${ }^{37}$. In fact, restrained eating behavior refers to the intention to restrict food intake, which is more likely to be under cognitive control than under internal or other physiological controls. When restrained eating habits break down, individuals tend to be disrupted or disinhibited by stimuli. Since people with greater restrained eating behavior showed larger increases in healthy food diversity, the dCBT program successfully restructured their cognitive frame, leading to healthier eating behavior.

In a previous study using dietary intervention, in contrast to the dCBT program, dietary restraint was a predictor of unfavorable weight loss $^{36}$. This also illustrates the importance of targeting cognitive components to improve healthy eating behavior during lifestyle interventions, especially for people with severe dietary restraint.

\section{Psychological features as mechanisms and IR as a consequence of improved healthy eating behavior}

This study is also the first to provide comprehensive insight combining psychological and physiological features into changes in healthy eating behavior. Our results confirmed that individuals with a greater decrease in body shape dissatisfaction showed greater increases in healthy food intake and diversity. Previous studies have concluded that body shape satisfaction is closely linked to lifestyle-related behaviors and changes in body weight ${ }^{38}$. There is a higher risk of engaging in health-damaging eating behaviors, such as excessive dietary restriction, for people with high levels of body shape dissatisfaction ${ }^{39}$. The results suggest that improvements in body shape satisfaction could be a mediator of improved healthy eating behavior.

In addition, the study reports that people with a greater decrease in IR presented greater increases in healthy food intake and diversity. It is obvious that IR is one of the major issues for many chronic diseases that require healthy lifestyle modification as a treatment ${ }^{40,41}$. As a part of lifestyle behavior, unhealthy eating behavior induces IR. Thus, there is no doubt that as eating behavior becomes healthier, IR improves. Based on our results, IR improvement can be seen as a consequence of healthy eating behavior. 
Healthy eating behavior also leads to weight loss ${ }^{26}$. These results also indicate that weight loss improves IR, suggesting that healthy IR may be the outcome of improved healthy eating behavior.

\section{Strengths and limitations}

This study has several firsts to its credit. First, this is the first RCT to validate the efficacy of dCBT through changes in healthy diet eating behaviors. Second, this is the first study to assess eating behavior using both direct observation and self-report methods, with the highest number of food items. Third, this is the first study to examine the mechanisms of changes in food intake, proportion, and diversity, with both psychological characteristics and IR. Notwithstanding these strengths, this study also has limitations. First, the participants were in their $20 \mathrm{~s}$ and $30 \mathrm{~s}$, limiting generalizability. Second, although eating behavior is a comprehensive behavior, the indices we used to measure it were limited to calorie intake, food proportion, and diversity. In future studies, it is recommended that other dimensional measures of behavior be included, such as latency to initiate eating behavior or inter-response time. Lastly, missing data is a known limitation, and mean imputation methods could lead to underestimation of the variance. However, since there were only a few missing values, the single imputation method would positively and efficiently present this numerical dataset, providing further insight into the mechanisms underlying the association between eating behavior and both psychological and biological conditions.

\section{Conclusion}

This study examined, for the first time, the role of psychological conditions and IR in eating behavior changes such as food intake, proportion, and diversity during lifestyle modification intervention. In addition, eating behaviors were assessed using two different methods: a buffet test-meal (laboratory setting) and a food diary app (real-world setting). Healthy diet eating behaviors were successfully promoted by the dCBT intervention. Finally, the study demonstrated physiological and psychological adjustments supporting the metabolic mechanisms and consequences related to healthy eating behavior in humans. Investigating eating behaviors using both objective and self-report methods and measurement of psychological and physiological indices will allow us to implement individualized treatment in obesity clinics.

\section{Declarations}

\section{Acknowledgments}

We would like to thank Youngin Kim, the chief executive manager of Noom, for supporting this study by sharing the company's advanced digital technologies.

\section{Ethics approval and consent to participate}

All participants provided written informed consent for participation in the study. The Institutional Review Board of the Seoul National University Hospital approved the consent documents, and the study was conducted according to the principles of ethical research practice outlined by the Declaration of Helsinki. The trial was registered with ClinicalTrials.gov NCT03465306 (retrieved September 18, 2017, https://register.clinicaltrials.gov/NCT03465306).

\section{Consent for publication}

Not applicable

\section{Availability of data and materials}

The datasets used and/or analyzed during the current study are available from the corresponding author upon reasonable request.

\section{Funding}

This study was supported by a grant from the National Research Foundation of Korea (NRF), funded by the Korean Government, Ministry of Science and ICT (MSIT) (No. NRF-2018R1A5A2025964). 


\section{Author contributions}

MK designed the conceptual framework of eating behavior and collected the data. MK, SP, and CJK analyzed and interpreted the data. HJC conceived the project and provided valuable insights when devising and visualizing the concept of this project. MK wrote the manuscript and edited its contents. HJC reviewed the manuscript. All authors approved the final version of the manuscript for submission.

\section{References}

1. Malek, S. H., Hopkins, D. P., Molloy, M. \& McGloin, T. The public health challenge of youth smoking in North Carolina: putting what we know into practice. N. C. Med. J. 63, 153-161(2002). PMID: 12181850

2. Maffeis, C. et al. Distribution of food intake as a risk factor for childhood obesity. Int. J. Obes. Relat. Metab. Disord. 24, 75-80 https://doi.org/10.1038/sj.ijo.0801088 (2000).

3. Cooper, A. J. et al. A prospective study of the association between quantity and variety of fruit and vegetable intake and incident type 2 diabetes. Diabetes Care. 35, 1293-1300 https://doi.org/10.2337/dc11-2388 (2012).

4. Drescher, L. S., Thiele, S. \& Mensink, G. B. A new index to measure healthy food diversity better reflects a healthy diet than traditional measures. J. Nutr. 137, 647-651 https://doi.org/10.1093/jn/137.3.647 (2007).

5. Mela, D. J. Determinants of food choice: relationships with obesity and weight control. Obes. Res. 9 (4), 249-255 https://doi.org/10.1038/oby.2001.127 (2001).

6. McCrory, M. A. et al. Dietary variety within food groups: association with energy intake and body fatness in men and women. Am. J. Clin. Nutr. 69, 440-447 https://doi.org/10.1093/ajcn/69.3.440 (1999).

7. Gibson, E. L. Emotional influences on food choice: sensory, physiological and psychological pathways. Physiol. Behav. 89, 53-61 https://doi.org/10.1016/j.physbeh.2006.01.024 (2006).

8. Rolls, B. J., Engell, D. \& Birch, L. L. Serving portion size influences 5-year-old but not 3-year-old children's food intakes. J. Am. Diet. Assoc. 100, 232-234 https://doi.org/10.1016/S0002-8223(00)00070-5 (2000).

9. Pliner, P. \& Mann, N. Influence of social norms and palatability on amount consumed and food choice. Appetite. 42, 227-237 https://doi.org/10.1016/j.appet.2003.12.001 (2004).

10. Houben, K., Dassen, F. C. \& Jansen, A. Taking control: working memory training in overweight individuals increases selfregulation of food intake. Appetite. 105, 567-574 https://doi.org/10.1016/j.appet.2016.06.029 (2016).

11. Verstuyf, J., Patrick, H., Vansteenkiste, M. \& Teixeira, P. J. Motivational dynamics of eating regulation: a self-determination theory perspective. Int. J. Behav. Nutr. Phys. Act. 9, 21 https://doi.org/10.1186/1479-5868-9-21 (2012).

12. Berthoud, H. R. Mind versus metabolism in the control of food intake and energy balance. Physiol. Behav. 81, 781-793 https://doi.org/10.1016/j.physbeh.2004.04.034 (2004).

13. Soni, A. C., Conroy, M. B., Mackey, R. H. \& Kuller, L. H. Ghrelin, leptin, adiponectin, and insulin levels and concurrent and future weight change in overweight, postmenopausal women. Menopause. 18, 296-301 https://doi.org/10.1097/gme.0b013e3181f2e611 (2011).

14. Kullmann, S. et al. Selective insulin resistance in homeostatic and cognitive control brain areas in overweight and obese adults. Diabetes Care. 38, 1044-1050 https://doi.org/10.2337/dc14-2319 (2015).

15. Mirabelli, M. et al. Mediterranean diet nutrients to turn the tide against insulin resistance and related diseases. Nutrients. 12, 1066 https://doi.org/10.3390/nu12041066 (2020).

16. O’Neil, P. M. et al. Randomized placebo-controlled clinical trial of lorcaserin for weight loss in type 2 diabetes mellitus: The BLOOM-DM study. Obesity (Silver Spring). 20, 1426-1436 https://doi.org/10.1038/oby.2012.66 (2012).

17. Ott, V., Benedict, C., Schultes, B., Born, J. \& Hallschmid, M. Intranasal administration of insulin to the brain impacts cognitive function and peripheral metabolism. Diabetes Obes. Metab. 14, 214-221 https://doi.org/10.1111/j.1463-1326.2011.01490.x (2012).

18. Rathnayake, K. M., Madushani, P. \& Silva, K. Use of dietary diversity score as a proxy indicator of nutrient adequacy of rural elderly people in Sri Lanka. BMC Res. Notes. 5, 469 https://doi.org/10.1186/1756-0500-5-469 (2012). 
19. Blundell, J. et al. Appetite control: methodological aspects of the evaluation of foods. Obes. Rev. 11, 251-270 https://doi.org/10.1111/j.1467-789X.2010.00714.x (2010).

20. Katkade, V. B., Sanders, K. N. \& Zou, K. H. Real world data: an opportunity to supplement existing evidence for the use of longestablished medicines in health care decision making. J. Multidiscip. Healthc. 11, 295-304 https://doi.org/10.2147/JMDH.S160029 (2018).

21. Lara, J., Taylor, M. A. \& Macdonald, I. A. Is ad libitum energy intake in overweight subjects reproducible in laboratory studies using the preload paradigm?. Eur. J. Clin. Nutr. 64, 1028-1031 https://doi.org/10.1038/ejcn.2010.104 (2010).

22. Nair, N. S. et al. Reproducibility of energy intake, gastric emptying, blood glucose, plasma insulin and cholecystokinin responses in healthy young males. Br. J. Nutr. 101, 1094-1102 https://doi.org/10.1017/S0007114508042372 (2009).

23. Robinson-O’Brien, R., Larson, N., Neumark-Sztainer, D., Hannan, P. \& Story, M. Characteristics and dietary patterns of adolescents who value eating locally grown, organic, nongenetically engineered, and nonprocessed food. J. Nutr. Educ. Behav. 41, 11-18 https://doi.org/10.1016/j.jneb.2008.03.007 (2009).

24. Wansink, B. Environmental factors that increase the food intake and consumption volume of unknowing consumers. Annu. Rev. Nutr. 24, 455-479 https://doi.org/10.1186/1479-5868-9-21 (2004).

25. Sharp, D. B. \& Allman-Farinelli, M. Feasibility and validity of mobile phones to assess dietary intake. Nutrition 30,12571266(2014). doi: 10.1016/j.nut.2014.02.020

26. Kim, M. et al. Multidimensional cognitive behavioral therapy for obesity applied by psychologists using a digital platform: open-label randomized controlled trial. JMIR mHealth uHealth. 8, e14817 https://doi.org/10.2196/14817 (2020).

27. Foster-Powell, K., Holt, S. H. \& Brand-Miller, J. C. International table of glycemic index and glycemic load values. Am. J. Clin. Nutr. 76, 5-56 https://doi.org/10.2337/dc08-1239 (2002).

28. Cooper, Z. et al. Testing a new cognitive behavioural treatment for obesity: a randomized controlled trial with three-year follow-up. Behav. Res. Ther. 48, 706-713 https://doi.org/10.1016/j.brat.2010.03.008 (2010).

29. Goodrick, G. K. \& Foreyt, J. P. Why treatments for obesity don't last. J. Am. Diet. Assoc. 91, 1243-1247(1991). PMID: 1918743

30. Weintraub, M. Long-term weight control study: conclusions. Clin. Pharmacol. Ther. 51, 642-646 https://doi.org/10.1038/clpt.1992.76 (1992).

31. Fearnbach, S. N., Thivel, D., Meyermann, K. \& Keller, K. L. Intake at a single, palatable buffet test meal is associated with total body fat and regional fat distribution in children. Appetite. 92, 233-239 https://doi.org/10.1016/j.appet.2015.05.036 (2015).

32. Sim, A. Y. \& Cheon, B. K. Influence of impending healthy food consumption on snacking: nudging vs. compensatory behaviour. Physiol. Behav. 198, 48-56 https://doi.org/10.1016/j.physbeh.2018.10.010 (2019).

33. Mayer, L. E., Schebendach, J., Bodell, L. P., Shingleton, R. M. \& Walsh, B. T. Eating behavior in anorexia nervosa: before and after treatment. Int. J. Eat. Disord. 45 (2), 290-293 https://doi.org/10.1002/eat.20924 (2012).

34. Chmurzynska, A., Mlodzik-Czyzewska, M. A., Malinowska, A. M., Czarnocinska, J. \& Wiebe, D. Use of a smartphone application can improve assessment of high-fat food consumption in overweight individuals. Nutrients. 10, 1692 https://doi.org/10.3390/nu10111692 (2018).

35. Fuller, N. R. et al. Comparison of an electronic versus traditional food diary for assessing dietary intake: a validation study. Obes. Res. Clin. Pract. 11, 647-654 https://doi.org/10.1016/j.orcp.2017.04.001 (2017).

36. Liu, X. et al. Predicting weight loss using psychological and behavioral factors: the POUNDS LOST trial. J. Clin. Endocrinol. Metab. 105 (4), 1274-1283 https://doi.org/10.1210/clinem/dgz236 (2020).

37. Johnson, F., Pratt, M. \& Wardle, J. Dietary restraint and self-regulation in eating behavior. Int. J. Obes. (Lond.). 36, 665-674 https://doi.org/10.1038/ijo.2011.156 (2012).

38. Anez, E. et al. Body image dissatisfaction, physical activity and screen-time in Spanish adolescents. J. Health Psychol. 23, 36-47 https://doi.org/10.1177/1359105316664134 (2018).

39. Christoph, M. J., Larson, N. I., Winkler, M. R., Wall, M. M. \& Neumark-Sztainer, D. Longitudinal trajectories and prevalence of meeting dietary guidelines during the transition from adolescence to young adulthood. Am. J. Clin. Nutr. 109, 656-664 https://doi.org/10.1093/ajcn/nqy333 (2019). 
40. Ehrampoush, E. et al. Association between dietary patterns with insulin resistance in an Iranian population. Clin. Nutr. ESPEN 36, 45-52(2020). doi: 0.1016/j.clnesp.2020.02.011

41. Kullmann, S. et al. Brain insulin sensitivity is linked to adiposity and body fat distribution. Nat. Commun. 11, 1841 https://doi.org/10.1038/s41467-020-15686-y (2020).

\section{Figures}

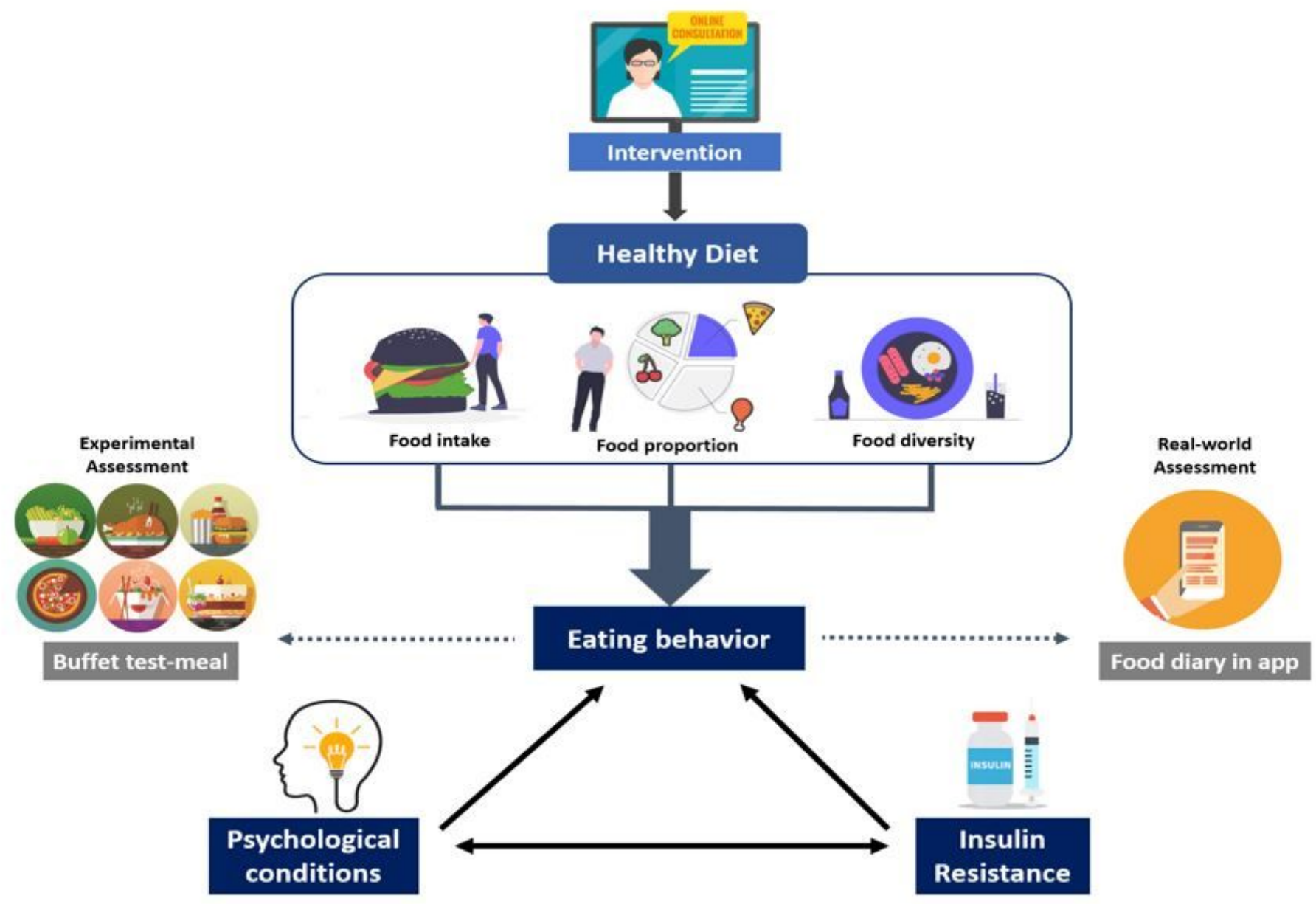

Figure 1

Conceptual framework of eating behavior with assessment methods and related factors. 

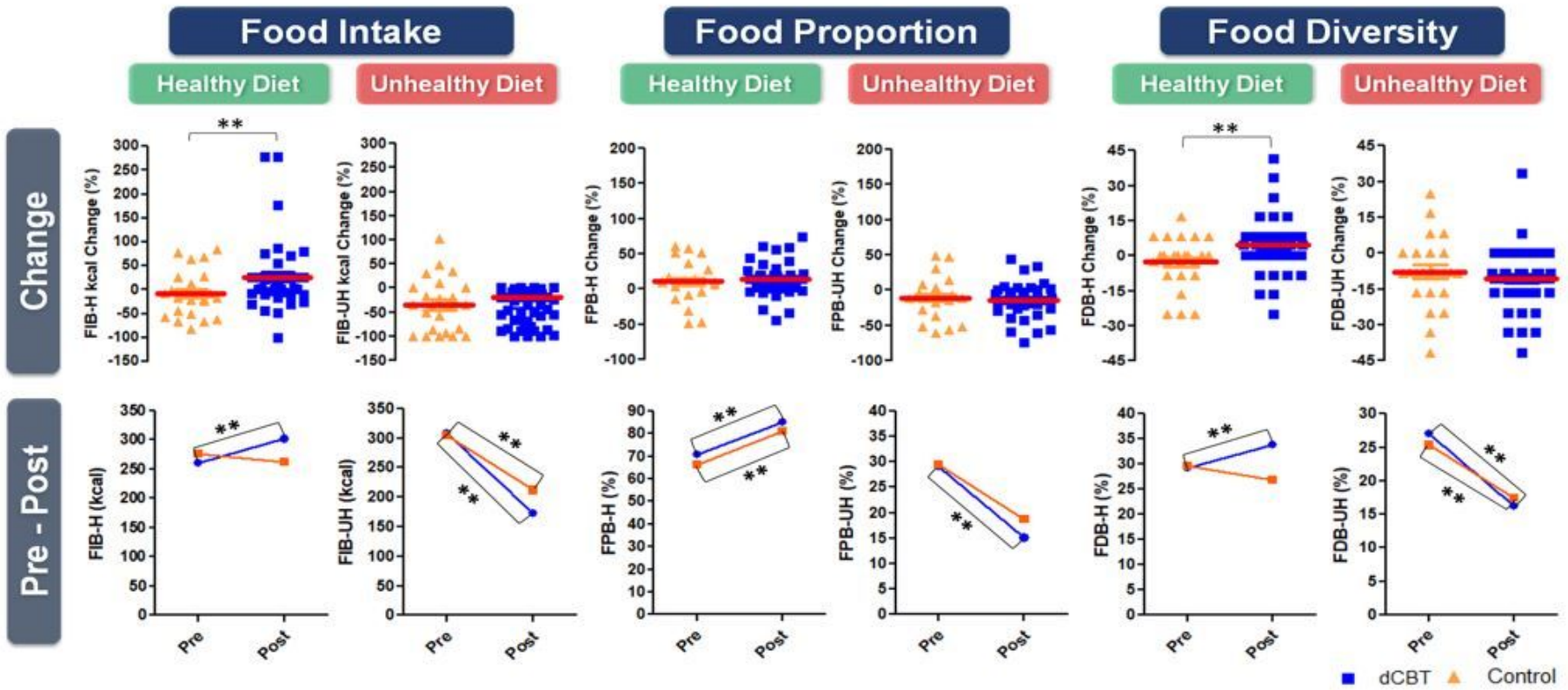

Figure 2

Overall results for each behavioral phenotype in both healthy and unhealthy diets from buffet test-meal assessment.
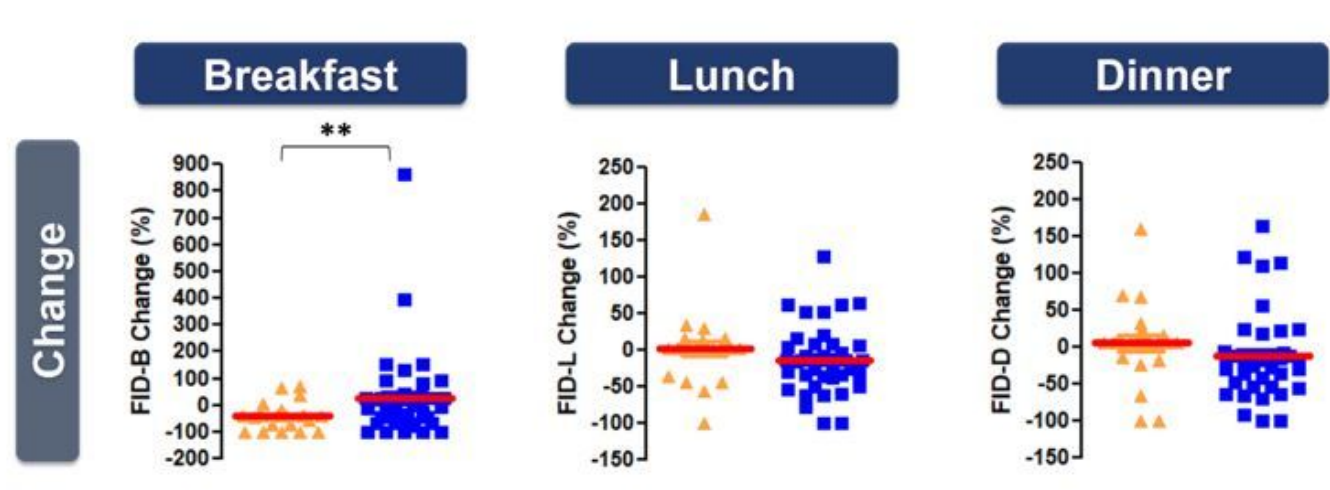

- dCBT a Control
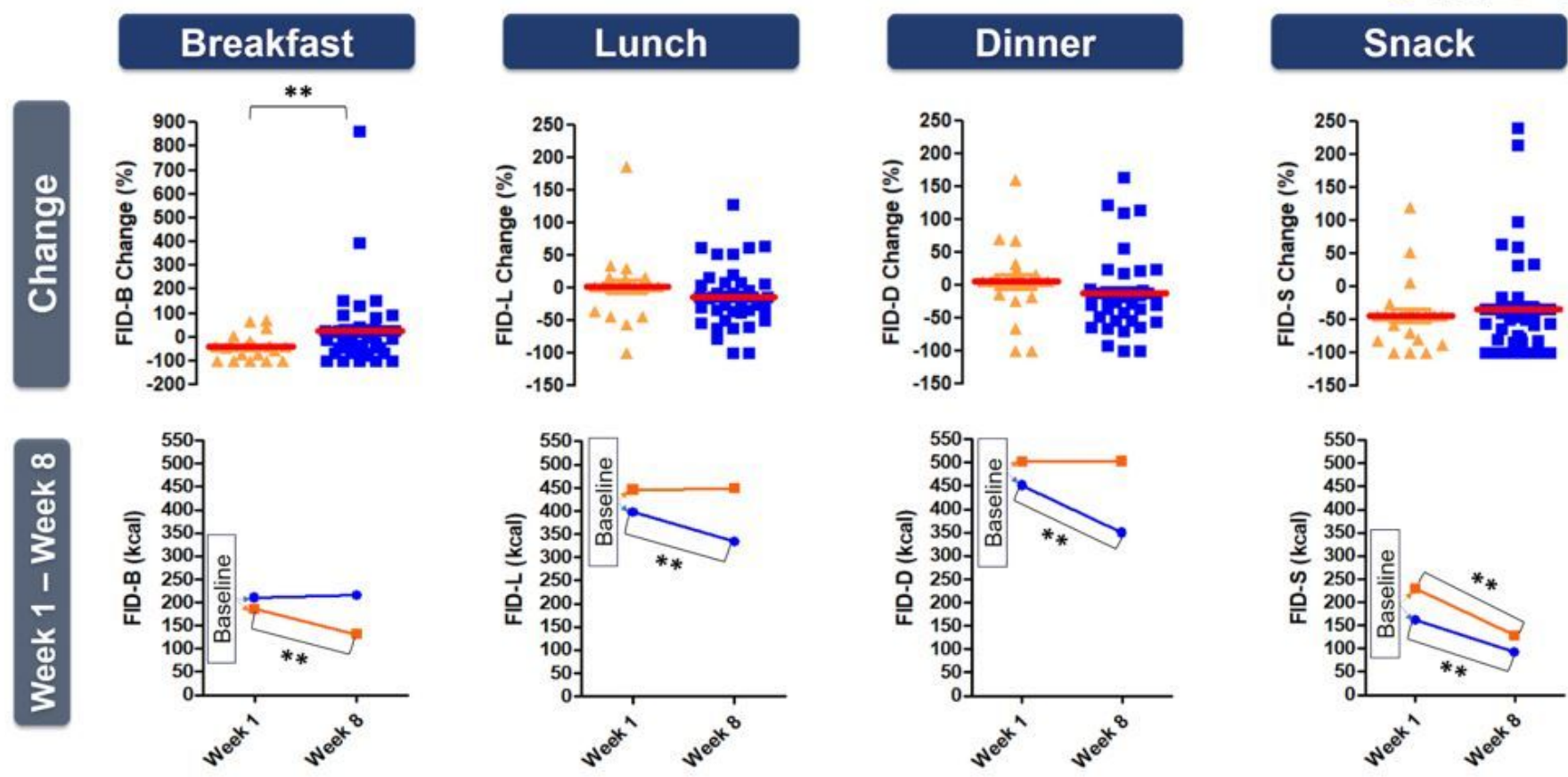

\section{Figure 3}

Overall results for the changes by food intake phenotype in each meal from the food diary app assessment. 
A.

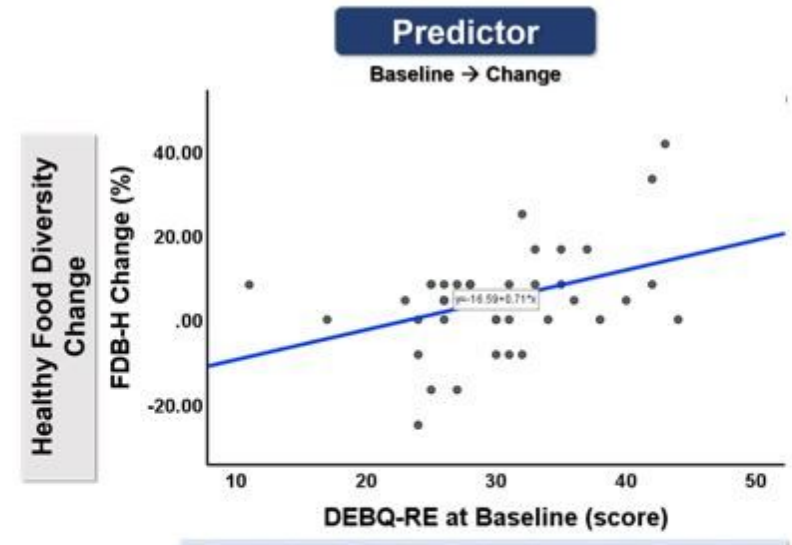

Restrained Eating Behavior at the Baseline

\section{Mechanisms}

B.

Psychological Change $\rightarrow$ Behavior Change

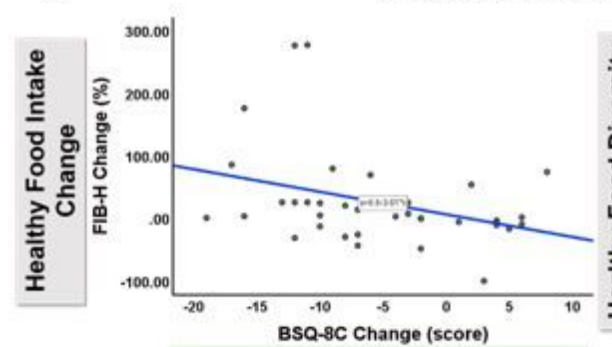

Body Shape Satisfaction Change

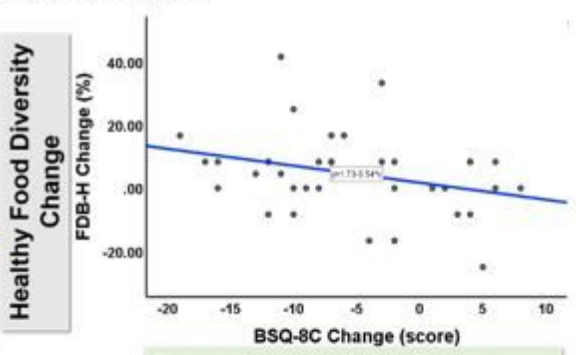

Body Shape Satisfaction Change

c.

\section{Consequence}
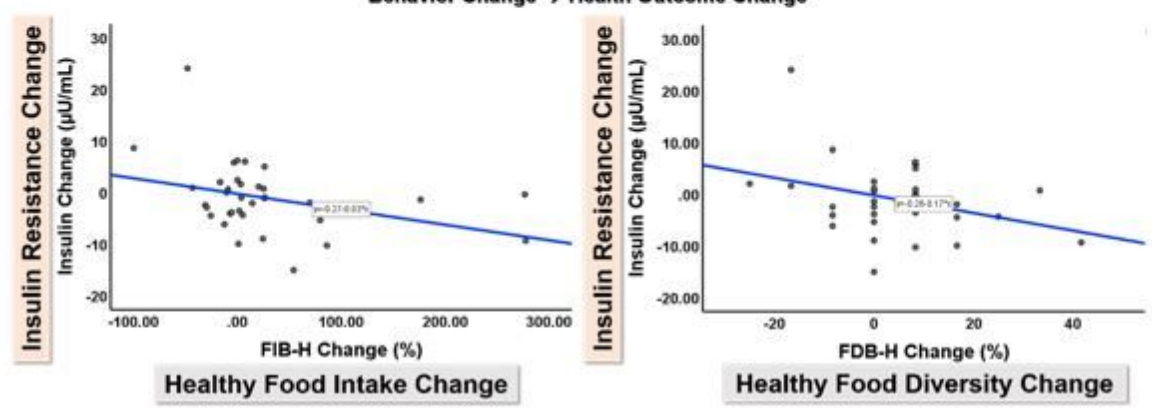

Figure 4

Predictive markers and mechanisms related to changes in major eating behavior phenotypes.

\section{Supplementary Files}

This is a list of supplementary files associated with this preprint. Click to download.

- Kimetal.HealthyEatingBehaviorChangewithPsychologicalFeaturesandInsulinResistanceBuffetandFoodDiaryAppAnalysis.docx 